\title{
PATRIA Y LEY EN LA CONSTRUCCION DE LA CIUDADANIA LOS LIBROS DE LECTURA Y LOS MANUALES DE INSTRUCCIÓN CÍVICA EN LA ESCUELA PRIMARIA ARGENTINA: 1870-1930
}

\author{
Silvina Gvirtz* \\ Gladys Barreyro**
}

\begin{abstract}
RESUMEN - El trabajo que se presenta analiza los libros de texto que tratan la problemática de la formación de la ciudadania en Argentina entre 1870 y 1930 . Se han analizado 39 textos de la época y se han comparado los mismos con los planes y programas de las escuelas dependientes del Consejo Nacional de Educación. El tema se encuentra presente tanto en los libros de lectura como en los manuales de instrucción civica. Sin embargo, en ambos tipos de texto, el tema es tratado diferenciadamente. Estos tipos de texto cumplen, en la lógica escolar, funciones diferentes. Mientras los libros de lectura procuran nacionalizar al inmigrante, los libros de instrucción civica procuran convertirlo en ciudadano. Esta doble función de los textos (nacionalizar y "ciudadanizar") no se refleja en los planes y programas de la época. Mientras estos v́ltimos observan una lógica disciplinar, en la que los contenidos de la formación cívica se incluyen en una materia especifica, y trabajan fundamentalmente temas vinculados a las leyes, los libros de texto dispersan los contenidos entre otras disciplinas y trabajan no sólo el tema de la ley sino además la formación cívica.
\end{abstract}

ABSTRACT - The paper presented here provides an analysis of the textbooks which deal with the question of the formation of citizenship in Argentina between 1870 and 1930. Thirty-nine textbooks of the epoch have been analyzed and compared with the plans and programs of schools under the jurisdiction of the National Education Council (Consejo Nacional de Educacion). The theme appears as much in the reading texts as in the civics instruction manuals. However, in each of these types of texts, the subject is dealt with a different way. These two types of texts play separate roles within the scholastic logic. While the reading texts strive to achieve nationalization of the inmigrants, the civics texts seek to convert them into citizens. This dual function of the textiooks (to nationalize and to create citizens) is not reflected in the plans and programs of the epoch. While the latter observe a disciplinary logic, where the elements of civic formation are included in a specific subject and work fundamentally with matters related to the law, the reading textbooks disperse the contents throughout other disciplines and not only deal

* Doctora en Educación y profesora adjunta del Centro de Formación e Investigación en Enseñanza de las Ciencias de la Universidad Nacional de Buenos Aires. Este artículo es parte de un proyecto de investigación sobre historia de las disciplinas escolares en Argentina entre 1870 y 1930, acreditado en la Universidad Nacional de Buenos Aires. La Dra. Gvirtz es profesora de dicha Universidad y es la directora del proyecto. La Lic. Barreyro es investigadora en el mismo.

** Licenciada en Letras.

\begin{tabular}{|l|l|l|l|l|l|} 
VERITAS & Porto Alegre & v. 43 & $\mathrm{n}^{\circ}$ especial & Dezembro 1998 & p. 173-182 \\
\hline
\end{tabular}


Los textos enfocan el tema desde la lógica de la dispersión.

PALABRAS LLAVE - Historia del curriculum, Instrucción cívica, libros de texto. with the subject of the law but also with the subject of the nation. The texts deal with these matters within the logic of dispersion.

KEY WORDS - History of the curriculum, instruction civic, text books.

\section{1 - Introducción}

En el marco de los procesos de su independencia politica, a partir de 1810, las Provincias Unidas del Río de la Plata ${ }^{1}$ intentan generar nuevos modos de educación más ajustados a los nuevos tiempos. Una de las preocupaciones de las élites gobernantes era la de hallar una modalidad que permitiese superar la dispersión que, en la época colonial, caracterizaba a la educación, especialmente a la educación elemental dirigida a la infancia (Narodowski, 1994). Hasta aquel entonces, la enseñanza de las primeras letras estaba en manos de muy diversos agentes.

Esta pretensión unificadora se concreta recién hacia fines del siglo XIX. Entre otros factores, la necesidad de unificar la diversidad provincial para competir como país agro-exportador en el mercado internacional, hace a la constitución del Estado Nacional Argentino. La construcción de un ejército único para todo el territorio, de una moneda única, de un gobierno único, de caminos transitables y comunicados en un territorio tradicionalmente fragmentado era un desafío no poco importante para esos tiempos.

Pero la Argentina, debía unificar también, a partir de su estado, una cultura que le permitiera constituirse como Nación. ${ }^{2}$ La segunda mitad del siglo XIX, se caracteriza por una importante llegada de contingentes migratorios provenientes de países tan distintos como España, Italia, Inglaterra, Alemania y Rusia. Buenos Aires, que se convierte a fines del siglo en la ciudad capital de la República Argentina, pasa de tener menos de 100.000 habitantes en 1850 a tener más de 500.000 en 1880. De este último total de habitantes, más de la mitad eran extranjeros. ${ }^{3}$ Orígenes diversos implicaban idiomas diferentes, conductas sociales diferentes, tradiciones diferentes. Para unificar esta diversidad cultural, en los valores de la modernidad, la escuela se presentaba como la institución ideal.

Si bien hubo leyes de obligatoriedad desde fines del siglo XVII, en distintas provincias que van a formar parte del Estado Argentino, la Ley 1.420 de educación primaria gratuita y obligatoria para todos, promulgada en 1884, se constituye en un hito, por ser una ley nacional con poder sobre todo el territorio argentino. La misma proclama una educación primaria no religiosa y estipula, en su artículo sexto, los contenidos mínimos de la enseñanza entre los que se destacan: lectura

1 La República Argentina constituye una parte del territorio de lo que fueron las Provincias Unidas del Río de La Plata.

2 Como se señala en el ya clásico trabajo de Oszlack O. (1985) La formación del Estado argentino "[...] la formación del estado nacional es el resultado de un proceso convergente, aunque no univoco, de constitución de una nación y un sistema de dominación" (p. 17). 
y escritura de la lengua castellana, aritmética, historia y geografía del teritorio argentino, idioma nacional y conocimiento de la Constitución Nacional para varones y mujeres.

Esta ley fue acompañada por la implementación de políticas tendientes a dos objetivos. Por un lado, se trataba de expandir cuantitativamente la escolaridad, de masificar y homogeneizar la escolarización elemental. Las políticas de escolarización implementadas en todo el territorio argentino lograron que en 1869 el de un 77,4\% de población argentina analfabeta en 1869, se reducia a un 35,9\% en 1914.

Por otro, las políticas promueven la formación de conductas y valores para una ciudadanía moderna. Disciplinar y homogeneizar la población, en función de Ios contenidos y conductas de la modernidad era, en este contexto, un problema de politica nacional. Para tal efecto, el Estado Nacional y las provincias regulaban no sólo los contenidos que se plasmaban en planes y programas sino también los contenidos de los libros de texto que se usaban en las escuelas. Es por este motivo que los libros debían ser aprobados por el Estado para luego poder ser utilizados en las escuelas.

Este trabajo versará sobre las estrategias de política educativa especificamente empleadas para con los libros de texto que se ocupaban de la formación del ciudadano, en la República Argentina, a partir del análisis de planes y programas y de 39 libros de texto utilizados entre 1870 y 1930.

\section{2 - La formación del ciudadano: libros de lectura y manuales de instrucción. Más allá de la disciplina escolar}

Una primera revisión de los planes y programas de la época muestra a la temática en cuestión encuadrada en la lógica de las disciplinas escolares. Los contenidos vinculados a la formación del ciudadano se encontraban en disciplinas tales como Instrucción Cívica ${ }^{4}$ o Moral, Urbanidad e Instrucción Cívica, ${ }^{5}$ según la época y el matiz predominantemente legalista o no que se le diera a los mismos. Una de las estrategias privilegiadas para la enseñanza fue el incluir y convertir estas temáticas en disciplina escolar.

El alcance de la misma era, sin duda, objeto de discusión. Los cambios frecuentes en los planes y programas dan cuenta de que la relación entre los contenidos de la instrucción cívica, la moral y la urbanidad no era simple. Sin embargo, frente a los tiempos más cambiantes de la política curricular, los libros de texto parecían operar con otra dinámica, diferente de la disciplinar y con otras duraciones, más lentas y más estables.

Cuando se procede a estudiar la temática en los libros de texto surgen dos cuestiones que no son menores. La primera es que las estrategias textuales de formación del ciudadano no respetaban aquel corte disciplinar de los planes y programas. Estas temáticas se presentaban, también y muy centralmente, como

4 Denominación dada a la materia en los planes de 1886/7. Monitor de la Educación Común, tomo VI, p. 397, Tomado de Aisenstein A. (1998) "Relevamiento de planes y programas de la República Argentina", CEFIEC, UNBA.

5 Denominación de la materia en los planes de 1901. Monitor de la Educación Común, tomo XVIr, p. 202, tomado de Aisenstein A. (1998, op. cit.). 
parte del contenido de los libros de lectura. Estos últimos estaban destinados a la enseñanza y práctica de la lectura en la escuela. Auxiliaban disciplinas curriculares como Lectura e Idioma Nacional. Trabajaban la lectura, en esta época, con contenidos vinculados al problema de la ciudadanía. Así, manuales, compendios y libros de instrucción cívica compartían con los libros de lectura la ardua tarea de contribuir a la formación del ciudadano. ${ }^{6}$ Sin embargo, unos y otros tipos de libro no se yuxtaponían, cumplían en la lógica escolar funciones diferenciadas.

En cuanto a sus características discursivas, los libros de lectura encontrados muestran un predominio de discurso literario. Los mismos contenían una selección de textos tales como poesías, algunos fragmentos de novelas costumbristas 0 históricas, fábulas, poemas y otros. Aquellas lecturas vinculadas con la formación del ciudadano eran centralmente emotivas. Esos textos mostraban elementos de la subjetividad del emisor, utilizando "subjetivemas"7 (adjetivos, sustantivos y verbos subjetivos) y estaban escritos en primera persona del plural, especialmente utilizando un "nosotros inclusivo": "Que nuestros corazones palpiten siempre por la patria, tan dificilmente definible y tan fácil de sentirse"g (Los subrayados son nuestros).

A diferencia de los libros de lectura, los manuales de instrucción cívica presentaban como característica principal el estar escritos utilizando discurso informativo de tipo legal. Se trataba de textos donde predominaba la tercera persona y se generaba por tanto la pretensión de "neutralidad y objetividad". En efecto, estos libros, en su mayor parte, transcribían artículos de leyes y, especialmente, los de la Constitución Nacional de la República Argentina ${ }^{10}$ :
"La Nación Argentina ha adoptado la forma de gobierno republicana, representativa, fede- ral, como lo establece el artículo 10. de la Constitución Nacional. [...] Se llaman ciudadanos argentinos todos los argentinos varones mayores de diez y ocho años, que gozan de los derechos politicos."11

Entre ambos tipos existía también un tipo mixto que recuperaba estrategias discursivas de uno y otro. Se trata de los llamados libros de lectura de instrucción moral y civica. Eran libros de lectura, auxiliares para la enseñanza de la disciplina "lengua", pero que ejercitaban el hábito de la lectura a partir de contenidos exclusivamente vinculados a la formación de la ciudadanía moderna. Estos libros utilizaban tanto el discurso emotivo como el informativo.

6 También los libros de historia eran utilizados en la formación de la ciudadanía aunque no serán tratados en este artículo. Para el abordaje de ese tema ver BRASLAVSKY (1992 y 1993).

7 "Unidades léxicas marcadas subjetivamente" (KERBRAT ORECCHIONI, 1986). Su utilización revela la subjetividad del emisor con respecto a su mensaje.

8 Kerbrat Orecchioni, op.cit.

9 COTTA, Juan Manuel. Lecturas morales para formar el carácter de los niños. Buenos Aires: Cabault, 1916.

10 Tanto era así que los organismos estatales encargados de controlar y supervisar el contenido de los libros de texto denuncian la afección a la copia de las leyes en los libros de instrucción cívica. En 1902, La Comisión Revisora de Textos de Instrucción Civica y Moral, observa esta característica: "se han concretado a copiar literalmente los artículos de la Constitución Nacional cometiendo toda clase de errores cuando se ha tratado de su comentario, de su fundamento legal de la apreciación y exposición de un hecho histórico" (p. 14).

11 GUERRINI. Francisco. El ciudadano argentino. Nociones de Instrucción, 1932. 
El ejemplo paradigmático de este último tipo es Cómo se ama a la patria de Ricardo Levene. En ese libro coexisten lecturas como "la familia argentina" donde ilustra la vida cotidiana familiar y en la que presenta fragmentos tales como:" $\mathrm{La}$ familia argentina ha poseido y posee bellas virtudes. Es la más característica la hospitalidad. El hogar argentino se abre generosamente al amor y a la caridad" con definiciones como: "La igualdad es el principio según el cual en la democracia argentina no se admiten prerrogativas de sangre ni de nacimiento".

De este modo, pueden distinguirse distintas estrategias discursivas para distintos tipos de textos que lejos de yuxtaponerse o parecer el resultado del azar, presentan regularidades significativas que conforman las estrategias textuales respecto de la formación del ciudadano.

Cuadro que cruza los tipos de libro con los tipos de discurso

\begin{tabular}{|l|c|c|}
\hline & $\begin{array}{c}\text { Discurso } \\
\text { informativo }\end{array}$ & Discurso emotivo \\
\hline Libro de lectura & $\mathrm{x}$ & $\mathrm{x}$ \\
\hline Manual de Instrucción Cívica & $\mathrm{x}$ & $\mathrm{x}$ \\
\hline $\begin{array}{l}\text { Libro de lectura de Instrucción } \\
\text { Moral y Civica }\end{array}$ & & \\
\hline
\end{tabular}

Puede señalarse que mientras en los primeros grados la formación cívica se realizaba exclusivamente a través de los libros de lectura y se apelaba básicamente a la emotividad del alumno, en los grados superiores la instrucción cívica tenía, además, una especificidad disciplinar y adquiría mayor relevancia el contenido informativo de los textos a partir del uso de los manuales..$^{12}$

Cabe agregar que los libros de Lectura en general estaban escritos por docentes mientras que los libros de Instrucción Cívica, aún cuando eran para nivel primario, estaban escritos, mayormente, por abogados..$^{13}$

\section{3 - Los temas de la formación del ciudadano: el Estado y la Nación a través de la ley y de la patria}

Puede afirmarse que en el periodo 1870-1930 la formación del ciudadano se plasma en los libros de texto a partir de dos ejes: ley y patria. El conocimiento de la ley permitiria la formación del sujeto incluido en una nación jurídicamente organizada pero, el conocimiento de ciertas nociones respecto de lo que es la patria le permitiría el acceso a una de las condiciones necesarias, aunque no suficientes para ser ciudadano: el manejo de ciertos ritos y tradición. La ley se asentaba, en los textos, sobre la idea de patria y nación.

12 Cabe señalar que el Reglamento para las escuelas comunes(1889) del Consejo Nacional de Educación indica en su art 33: "En los dos primeros grados no se permitirá otro texto que el de lectura". Por su parte, Ana Blasco de Selva (en El nuevo lector argentino. Libro de lectura para 4º año, 1910) señala en su prólogo: "Considerando que, de acuerdo con una excelente práctica y disposiciones reglamentarias vigentes, los cursos infantiles no usan más libro que el de lectura [...]".

13 Cuando estos textos no están escritos por hombres de leyes, se pudo constatar que una nota de algún abogado que avala el contenido del texto. 
Ahora bien, mientras los manuales de instrucción cívica privilegian las temáticas vinculadas a la ley en primer lugar y a la patria en segundo, los libros de lectura hacen mayor cantidad de referencias a la temática patriótica, la ley aparece en menor medida.

Cuadro que cruza los temas tratados en los distintos tipos de libros escolares

\begin{tabular}{|l|c|c|}
\hline & Ley & Patria \\
\hline Libro de lectura & & $\mathrm{x}$ \\
\hline Manual de Instrucción Cívica & $\mathrm{x}$ & \\
\hline $\begin{array}{l}\text { Libro de lectura de Instrucción } \\
\text { Moral y Cívica }\end{array}$ & $\mathrm{x}$ & $\mathrm{x}$ \\
\hline
\end{tabular}

En los manuales de instrucción cívica la patria es equivalente al concepto de Nación:

"La nación es un conjunto de hombres que reconocen la misma autoridad, idioma y costumbres."

"La lamamos nuestra patria, no solamente porque es el país o nación donde hemos nacido, sino porque aquí tenemos nuestros padres, nuestros compañeros de escueia y nuestros compatriotas, hablamos todos el mismo idioma, obedecemos las mismas leyes, tenemos los mismos deberes y derechos." 14

En los libros de lectura, en cambio, los modos básicos en que aparece la mención de la patria son:

a) a partir del relato del mito de los orígenes de la República Argentina,

b) a partir de la incorporación de próceres como "modelos ejemplares".

a) con este procedimiento que aparece tanto en los libros de instrucción cívica como en los de lectura la visión que de la historia argentina se presenta es una mitificación de la historia ${ }^{15}$ que consiste en la creación de una épica patriótica.

b) Los modelos ejemplares eran un subgénero muy utilizado en el siglo XIX en la literatura infantil mundial. Los libros de lectura relevados desde 1870 incluyen lecturas sobre las vidas de personas como Franklin, los Curie, Miguel Angel, Washington con sus biografías, anécdotas de su vida, aportes a la humanidad en las cuales se destaca el tono moralizante de los relatos. Este univeŕsalismo ya incluye, en la ultima década del siglo XIX, a los próceres históricos como modelos a imitar.

En los libros de lectura aparecen los modelos ejemplares universales con una función moralizante promoviendo conductas. A partir de 1910, estos modelos son sólo los "patriotas". Hacia 1930 se puede comprobar una vuelta a la inclusión de modelos más universalistas. ${ }^{16}$

14 Guerrini, op. cit.

15 Braslavsky, 1993.

16 Aproximadamente en 1910 en el campo intelectual argentino, con el Centenario de la Revolución de Mayo, surge la que se ha dado en llamar "generación del centenario"un movimiento cultural que ve en el advenimiento masivo de la inmigración una pérdida de identidad nacional. Implica una vuelta a las tradiciones rescatando el folklore provinciano, un hispanismo nostálgico y una revalorización del idioma, entre otras cosas (Paya y Cardenas, 1978; Sarlo, 1983). 
En lo que respecta a la ley, los manuales de instrucción cívica de fines del siglo XIX se caracterizan por difundir y hacer conocer la Constitución Nacional. Este era un contenido obligatorio para todas las escuelas desde la sanción, en 1884, de la Ley 1420 de enseñanza obligatoria. Aún cuando en 1890 se lo sustituyó por la enseñanza de "Instrucción Cívica", se pudo corroborar que en muchos manuales de Instrucción Cívica se lo siguió incluyendo: "La organización del gobierno y los tres poderes", "los derechos y deberes de los ciudadanos y habitantes", son tópicos constitutivos de estos manuales. Sin embargo, entre los deberes y derechos de la ciudadanía, se enfatizan algunos en detrimento de otros. Entre los relevados se destacan: "el servicio militar", "la obligatoriedad escolar" y, a partir de 1912, cuando en Argentina se sanciona la Ley Saenz Peña de voto secreto y obligatorio, en los textos comienza a plantearse el tema del "sufragio universal".

Por ejemplo, en Educación civica primaria, Juan Beltrán dedica 14 páginas al tema del sufragio. explicando muy detalladamente qué es, quiénes votan, qué es un comicio, lo que es una libreta civica y las consecuencias de no votar a un buen candidato.

Con respecto al servicio militar, en otro manual se señala que: "Todo ciudadano argentino debe prestar el servicio militar personal. [...] El primer acto que debe practicar todo ciudadano argentino para prestar el servicio de las armas se llama enrolamiento."17

En el mismo libro, además se informa acerca del ejército de la Nación, sus divisiones, la excepción al servicio militar, el juramento a la bandera, vinculando estas temáticas con la obligatoriedad del servicio militar. También se aportan fotografías del Juramento a la Bandera, de una Brigada de Infantería y del Colegio Militar de la Nación.

El tema de la obligatoriedad escolar está presente en la mayoría de los libros. v.g., en uno de ellos se presenta la siguiente leyenda:

"Qué felices son los niños que van a la escuela. La ley castiga con multas a los padres, tutores o cualquier persona que teniendo niños a su cuidado o servicio no cumpla esta obligación. La instrucción es obligatoria y gratuita. El Consejo Nacional de Educación y los Consejos Escclares de las provincias dan matriculas, libros y útiles a los niños pobres."18

En este contexto, los manuales de instrucción civica destacan el problema de la inmigración, a partir del espacio brindado a la distinción entre ciudadano y habitante. Los textos presentan una mirada integracionista y práctica: se trataba de convertir al inmigrante en ciudadano. Al respecto cabe destacarse la aparición en algunos de los libros del formulario para solicitar la ciudadanía argentina. ${ }^{19}$

17 Guerrini, op. cit.

18 BELTRAN, J. Educación civica primaria. Buenos Aires: Librería e Imprenta Europea, 1916.

19 MORAN, V. Instrucción cívica y moral. Buenos Aires: Moly y Lasserre, 1938. 


\section{4- Conclusiones}

Las estrategias textuales de las politicas educativas para la construcción de la ciudadanía y las estrategias curriculares (es decir la delimitación y selección de contenidos en planes y programas de la época) no tienen una correspondencia biunívoca. Mientras la lógica curricular ciñe la Instrucción Cívica a la lógica de las disciplinas escolares, los libros de texto dispersan el tratamiento del tema y lo contemplan en libros explícitamente destinados a otras disciplinas tales como Lengua o Historia. La producción significativa en la época de libros de lectura de instrucción moral y cívica, escritos por prestigiosos autores (v.g. Ricardo Levene) está indicando el lugar de esta problemática en el contexto de las políticas sobre la producción y contenido de los textos.

Pero además, mientras la lógica curricular limita el contenido a los últimos años de la escuela primaria, los libros de lectura la trabajan ya en los primeros grados, a partir de estrategias bien específicas. En sintesis pude decirse que para encarar el estudio de la historia del curriculum o de los contenidos escolares, o aún para encarar el estudio de la historia de las políticas curriculares, por lo menos en la Argentina, no puede pensarse en la lógica de la correspondencia (los libros responden a la lógica estipulada en planes y programas) ni en una lógica gestáltica que permitiría inferir los contenidos a partir de mirar sólo textos o sólo planes y programas. Los modos de relación entre los diversos niveles curriculares parecen ser bastante complejos y merecen, por tanto, ser cuidadosamente investigados.

\section{Bibliografia}

APPLE, M. Maestros y textos. Barcelona: Paidós, 1989.

A.A.V.V. "Educación cívica e histórica en los libros de texto". En: Propuesta Educativa, Buenos Aires, Flacso-Miño y Dávila, n. 8, 1993.

A.A.V.V. "Los usos de la historia en los libros de texto". En: Propuesta Educativa, Buenos Aires, Flacso-Miño y Dávila, n. 7, 1992.

BERTONI, Lilia. “Construir la nacionalidad. Héroes, estatuas y fiestas patrias, 1887-1891". En: Boletín del Instituto de historia argentina y americana "Dr. E. Ravignani", Tercera serie, n. 5, 1992.

BRASLAVSKY, C. Los usos de la historia en la educación argentina. con especial referencia a los libros de texto para las escuelas primarias. 1853-1916. FLACSO, 1992. Serie de documentos e informes de investigación.

- Los usos de la historia en los libros de texto para las escuelas primarias argentinas. 19161930. FLACSO, 1993. Serie de documentos e informes de investigación.

DEVOTO, F. "Idea de Nación, inmigración y cuestión social en la historiografia académica y en los libros de texto de Argentina 1912-1974". En: Propuesta educativa. Buenos Aires, As. Flacso-Miño y Dávila, n. 8, 1993.

GARCIA CRESPO, Clementina. Léxico e ideologia en los libros de lectura de la escuela primaria. (1940-1975). Ediciones Universidad de Salamanca. Instituto de Ciencias de la Educación, 1983.

HALPERIN DONGHI, T. Historia Contemporánea de América Latina. Ed. Alianza, 1981.

PAYA, C., CARDENAS, E. El primer nacionalismo argentino. Buenos Aires: Peña Lillo, 1978.

PLOTKIN, Mariano. Mañana es San Perón. Propaganda, rituales políticos y educación en el régimen peronista (1946-1955). Buenos Aires: Ariel, 1994.

SARLO B. "La argentina del centenario: campo intelectual, vida literaria y temas ideológicos". En: Ensayos Argentinos, Buenos Aires, Centro Editor de América Latina, 1983.

VARELA, Mirta. Los hombres ilustres del Billiken. Héroes en los medios y en la escuela. Buenos Aires: Colihue, 1994. 
WAINERMAN, C., BARCK DE RAIJMAN, R. Sexismo en los libros de lectura de la escuela primaria. Buenos Aires, IDES, 1987.

WAINERMAN, C., HEREDIA, M. (s/f) "Los libros de lectura a las puertas del siglo XXI: género, trabajo y familia." (mimeo).

\section{Listado de textos relevados}

ANDRES de VALS, J. Cantos rodados. Buenos Aires: Crespillo. (s/f)

ARENA, Luis. Yunque sonoro. 1. ed. Buenos Aires: Estrada, 1942.

AUBIN, J. Vida diáfana. Buenos Aires: Estrada, 1913.

BEAUDOIN. L. Lecturas para 6o. grado. Buenos Aires: Talleres Gráficos Argentinos, 1929. L. J. ROSSO.

BELTRAN, J. Educación civica primania. Buenos Aires: Libreria e Imprenta Europea, 1916.

BERDIALES, G., INCHAUSPE, P. Nuevo mundo. Lecturas para quinto grado. Iniciación Literaria. Buenos Aires, 1941.

BERDIALES, Germán. Lecturas para la niña que se hace mujer. 3. ed. 1953.

BERRUTI, José J. Lecturas morales e instructivas. Buenos Aires: 42. ed. Estrada. (s/f)

BLASCO DE SELVA, A. El nuevo lector argentino. Libro de lectura para $4^{2}$ año. 1910.

BLOMBERG, Héctor Pedro. El sembrador. 15. ed. Libro de lectura para $3^{\text {er }}$ grado. Angel Estrada Editores. (s/f)

BOURGUET,L. Panoramas. Texto de lectura para $4^{\complement}$ grado. Buenos Aires: Independencia, 1929.

BRAÑA DE IACOBUCCI, B., IACOBUCCI, Guillermo. Fuentes de vida. Libro de lectura para 6o grado. Buenos Aires Kapelusz, 1937..

CONDOMI ALCORTA, Arturo. Compendio de instrucción cívica. Buenos Aires: Cabault, 1928.

COTTA, Juan Manuel. Lecturas morales para formar el carácter de los niños. Buenos Aires: Cabault, 1916.

COTTA, Juan Manuel. El compañero espiritual. Libro de lectura para $6^{0}$ grado. Buenos Aires: F. Crespillo Ed., 1933.

COTTA, Juan Manuel. Evangelio escolar (Para los niños inteligentes y los maestros consagrados). Imprenta Frascoli y Bindi. (s/f

DOUMER. EI perfecto ciudadano. Barcelona, Parera, 1915.

EIZAGUIRRE, J. La patria. Elementos para estimular en el niño argentino el amor a la patria y el respeto a las tradiciones nacionales. Buenos Aires: Pedro Igón y Cía., 1894.

Ejemplos morales o sea Lectura para niños. París-México: Bibliotecâ de la Juventud, 1898. (Sinmención de autor)

ESTRADA, T. Lecturas argentinas. Buenos Aires: Estrada, 1908.

FERNANDEZ ALONSO, Eloy. El argentino. 20. ed. Antologia de autores argentinos para $5^{\circ}$ y $6^{\circ}$ grados. Buenos Aires, 1936.

KAPELUSZ, FERREYRA, A., AUBIN, J. El nene. $2^{\circ}$ Libro. Buenos Aires: Estrada, 1907.

FIGUERA, J. Un buen amigo. Buenos Aires Cabault, 1916.

FRAGUEIRO, C: L. Vidas de argentinos ilustres. Texto de lectura para las escuelas. Buenos Aires: Igón, 1884 .

FREYRE DE JAIMES, C. Ameno y útil. Buenos Aires: Estrada, 1910.

Gobierno propio. Buenos Aires: Cabaut, 1933.

GORRITI, J. M. Perfiles. Buenos Aires: Lajouane, 1892.

GUERRA, Rosa. Julia o la educación. Libro de lectura para las niñas. Buenos Aires: Imprenta de "El Mercurio", 1863.

GUERRINI. Francisco. El ciudadano argentino. Nociones de Instrucción Cívica. La Plata: Olivieri y Domínguez, 1932.

LAMADRID, E. Nociones prácticas de moral. Buenos Aires: Féiix Lajouane, 1894.

Lecciones cortas de moral. 1915. (sin mención de autor)

LEVENE, Ricardo. Como se ama a la patria. Buenos Aires: Aquilino Fernández Editor, 1912.

LOPEZ DE NELSON, Emestina. Nuestra tierra. Buenos Aires: Coni. (s/f).

MESTORINO, O. Apuntes de instrucción moral y cívica. Buenos Aires: Estrada, 1936. 
MORAN, V. Instrucción civica y moral. Buencs Aires: Moly y Lasserre, 1938.

ORTEGA, Enrique. Compendio de instrucción cívica para uso de las escuelas. 4. ed. Buenos Aires: Pedro İgon y Cía Editores, Librería del Colegio, 1890.

RIVAROLA, Rodolfo. Educación moral y cívica. (1. ed. 1917). Buenos Aires: Kapelusz. 1940.

SALZA, Emilia. El derecho constitucional en manos de los niños. Buenos Aires: Cabaut, 1906.

WAGNER SOSA, P. Patria, hogar y fraternidad. Lecturas morales para $3^{\circ}$ y $4^{\circ}$ grado. Buenos Aires: Estrada, 1910. 